\title{
Application of Shuffled Frog Leaping Algorithm to Long Term Generation Expansion Planning
}

\author{
M. Jadidoleslam, E. Bijami, N. Amiri, A. Ebrahimi, and J. Askari
}

\begin{abstract}
This paper presents the application of an efficient shuffled frog leaping algorithm (SFLA) to solve the optimal generation expansion planning (GEP) problem. The SFLA is a meta-heuristic search method inspired by natural memetics. It combines the advantages of both genetic-based memetic algorithms and social behavior based algorithm of particle swarm optimization. Least-cost GEP is concerned with a highly constrained non-linear discrete dynamic optimization problem. In this paper the proposed formulation of problem, determines the optimal investment plan for adding power plants over a planning horizon to meet the demand criteria, fuel mix ratio, and the reliability criteria. To test the proposed SFLA method, it is simulated for two test systems in a time horizon of 10 and 20 years respectively. The obtained results show that compared to the traditional methods, the SFLA method can provide better solutions for the GEP problem, especially for a longer time horizon.
\end{abstract}

Index Terms-Generation expansion planning, probabilistic production simulation, shuffled frog leaping algorithm.

\section{INTRODUCTION}

The Generation Expansion Planning (GEP) is a problem to determine when, where, what type and how much capacity of new power plants should be constructed over a long-term planning horizon to meet forecasted demand according to a pre-specified reliability criteria. GEP is an important decision-making activity for utility companies. The main objective of GEP is to minimize the total investment, operating, and outage (energy-not-served) costs of power system.

There are generally two deterministic and stochastic approaches to solve the GEP problem. The Stochastic approach takes into account uncertainties associated with the input data, such as forecasted demand, fuel prices, economic and technical characteristics of new evolving generating technologies, construction lead times, and governmental regulations [1]. The deterministic approach solves the problem under different scenarios. In this case a fast and efficient method is required because of great number of simulations needed to be run under different scenarios.

Least-cost GEP is concerned with a highly constrained non-linear discrete dynamic optimization problem. The high non-linearity of a GEP problem originates from the nature of the production cost and the set of non-linear constraints. The dynamic programming (DP) approach is one of the most used

Manuscript received February 20, 2012; revised March 16, 2012.

M. Jadidoleslam, E. Bijami, N. Amiri, A. Ebrahimi, and J. Askari are with the Department of Electrical and Computer Engineering, Isfahan University of Technology, Isfahan 84156, Iran (e-mail: \{m.jadidoleslam, e.bijami,n.amiri\}@ec.iut.ac.ir, \{ebrahimi, j-askari\}@cc.iut.ac.ir). algorithms in GEP. However in the generation expansion problem, due to high dimensionality, the DP is not an efficient method for real power systems. In commercial packages like WASP [2], to overcome this difficulty, heuristic tunnel-based techniques are used in DP routine, where users pre-specified configurations and successively modified tunnels are considered to arrive at local optimums.

The emerging optimization techniques used to solve GEP problem were reviewed in [3]. In [4] the meta-heuristic techniques; such as Genetic Algorithm, Differential Evolution, Evolutionary Programming, Evolutionary Strategy, Ant Colony Optimization, Particle Swarm Optimization, Tabu Search, Simulated Annealing, and Hybrid Approach, are applied to solve GEP problem and are compared with DP. The results of this research show that drawbacks of DP can be overcome by these meta-heuristic techniques. In [5] an improved genetic algorithm (IGA) with stochastic crossover technique and elitism are applied to solve the GEP problem. The results of the IGA are compared with those of the conventional; such as simple genetic algorithm, the full DP and the tunnel-constrained DP.

The SFLA is a novel meta-heuristic optimization method inspired from the natural memetic evolution of a group of frogs when searching for the location that has the maximum amount of available food [6]. The SFLA combines the advantages of both genetic-based memetic algorithms and social behavior based particle swarm optimization algorithms. Recently, global optimization techniques using SFLA has been successfully applied to various areas of power system analysis such as unit commitment [7], dynamic optimal power flow [8], and optimal reactive power flow [9] problems.

Because of a high capability of SFLA to solve large scale optimization problems, in this paper it used to solve the GEP problem. Also, the efficiency of the SFLA is improved by applying the integer encoding of solutions, mapping procedure and penalty factor approach. Since in [5], [10], [11], the priority of GA in solving GEP problem has been proved, GA is applied and the obtained results by the GA are compared with those obtained by the SFLA.

This paper is organized as follows: in section II the proposed GEP formulation is given. An overview of the SFLA and solution methodology is described in section III. Section VI describes case studies and provides test results, and section $\mathrm{V}$ concludes the paper.

\section{GEP PROBLEM FoRMULATION}

Solving a least-cost GEP problem is equivalent to determine the optimum expansion plan over planning period 
that minimizes total cost including investment, operation, and, outage (energy-not-served) costs under several constraints introduced as follows.

\section{A. Cost Function}

Similar to WASP mathematical formulation [2], the cost (objective) function of the GEP problem can be represented by the following expression:

$$
\min C=\sum_{t=1}^{T}\left[\bar{I}\left(U_{t}\right)+\bar{M}\left(X_{t}\right)+\bar{O}\left(X_{t}\right)-\bar{S}\left(U_{t}\right)\right]
$$

where, $C$ is the total cost $(\$), t$ is the time stage $(1,2, \ldots, \mathrm{T}), T$ is the length of the study period (total number of stages), $U_{t}$ is the capacity vector of all candidate unit types in stage $t(\mathrm{MW})$, $X_{t}$ is the cumulative capacity vector of all existing and candidate units in stage $t(\mathrm{MW})$, and

$$
X_{t}=X_{t-1}+U_{t}
$$

The bar over the symbols has the meaning of discounted values to a reference date at a given discount rate $i$. Equation (2) is state equation for dynamic planning problem.

In order to calculate the present values of the cost components of (1), it is assumed that the capital investment for a candidate unit added by the expansion plan is made at the beginning of the stage in which it goes into service. Salvage value is assumed to occur at the end of the planning horizon. All the other costs including fix and variable, operation and maintenance (O\&M) costs, and outage cost are assumed to occur in the middle of the corresponding year. Therefore, the cost components of (1) are calculated as follow:

$$
\begin{gathered}
\bar{I}\left(U_{t}\right)=(1+i)^{-t^{\prime}} \times \sum_{k=1}^{N}\left[C I_{k} \times U_{t, k}\right] . \\
\bar{M}\left(X_{t}\right)=\sum_{y=0}^{s-1}\left[(1+i)^{-\left(t^{\prime}+0.5+y\right)}\right. \\
\left.\times \sum_{k=1}^{N}\left[F O M_{k} \times X_{t, k}+V O M_{k} \times G_{t, k}\right]\right] . \\
\bar{O}\left(X_{t}\right)=\sum_{y=0}^{s-1}\left[(1+i)^{-\left(t^{\prime}+0.5+y\right)} \times E E N S_{t} \times C E E N S\right] \\
\bar{S}\left(U_{t}\right)=(1+i)^{-T^{\prime}} \times \sum_{k=1}^{N}\left[\delta_{k, t} \times C I_{k} \times U_{t, k}\right]
\end{gathered}
$$

where

$$
\begin{array}{r}
t^{\prime}=t_{0}+s \times(t-1) . \\
T^{\prime}=t_{0}+s \times T .
\end{array}
$$

and

s number of years in each stage;

$\mathrm{t} 0$ number of years between the reference date for discounting and the first year of study;

$\bar{I}\left(U_{t}\right)$ present value of investment cost of the candidate units at the stage $\mathrm{t}, \$$;

CIk capital investment cost of kth unit, \$;

$\mathrm{Ut}, \mathrm{k}$ capacity vector of candidate units of type $\mathrm{k}$ in stage $\mathrm{t}$,
MW;

$\mathrm{N}$ total number of different types of units.

$\bar{M}\left(X_{t}\right)$ present value of total fix and variable, O\&M cost of existing and candidate units in stage $t, \$$;

$y$ variable used to indicate that the operation and maintenance $(O \& M)$ cost is calculated at the middle of each year;

FOMk fixed O\&M cost of kth unit, \$/MW;

$\mathrm{Xt}, \mathrm{k}$ cumulative capacity vector of units of type $\mathrm{k}$ in stage $\mathrm{t}$, $\mathrm{MW}$;

VOMk variable O\&M cost of kth unit, \$/MWh;

$\mathrm{Gt}, \mathrm{k}$ expected energy produced by kth unit in stage $\mathrm{t}$, MWh;

$\bar{O}\left(X_{t}\right) \quad$ present value of outage cost of the existing and candidate units, $\$$

EENS expected energy not served, MWh;

CEENS cost of EENS, \$;

$\bar{S}\left(U_{t}\right)$ present value of salvage value of the candidate units in stage $\mathrm{t}, \$$;

$\delta \mathrm{t}, \mathrm{k}$ salvage factor of $\mathrm{kth}$ unit added in stage $\mathrm{t}$.

\section{B. Constraints}

In this paper four types of defined constraints are represented by the following expressions:

$$
\begin{gathered}
0 \leq U_{t} \leq U_{\max , t} \\
\left(1+R_{\min }\right) \times D_{t} \leq \sum_{k=1}^{N} X_{t, k} \leq\left(1+R_{\max }\right) \times D_{t} \\
M_{\min }^{j} \leq \frac{X_{t, j}}{\sum_{k=1}^{N} X_{t, k}} \leq M_{\max }^{j} \\
\operatorname{LOLP}\left(X_{t}\right) \leq \varepsilon
\end{gathered}
$$

Equation (9) reflects the construction capability limits in each stage, in which $U_{\max , t}$ is the maximum construction capacity of units at stage $t$. Equation (10) presents reserve margin constraint in which $D_{t}$ is the peak demand in the stage $t$, (MW). Installed capacity in each stage, must lie between the given minimum and maximum reserve margins that represented by $R_{\min }$ and $R_{\max }$, respectively. The capacity mixes by fuel types are considered in (11) in which $M_{\min }^{j}$ and $M_{\max }^{j}$ are lower and upper bounds of $j$ th fuel type in stage $t$. Equation (12) is reliability criterion of the power system where $\varepsilon$ is the reliability criterion for maximum value of loss of load probability (LOLP).

Probabilistic production simulation is done using the Equivalent Energy Function (EEF) method [12]. Simulating production system at each stage of planning horizon calculates the expected energy produced by each unit in every stage of planning $\left(G_{t, k}\right)$ to be used in variable O\&M cost calculation of (4). The reliability criterion of loss of load probability (LOLP), used in (12), and the expected energy not served (EENS) used in the outage cost calculation by (5) are also determined by the used simulation procedure. 


\section{IMPLEMENTATION OF SFLA TO SOLVE THE LEAST COST GEP PROBLEM}

\section{A. An Overview of SFLA}

The SFL algorithm is a memetic meta-heuristic method that is derived from a virtual population of frogs in which each frog represents a set of feasible solutions. Each frog is distributed to a different subset of the whole population described as memeplexes. The different memeplexes are considered as different culture of frogs that are positioned at different places in the solution space (i.e. global search). A simultaneous independent deep local search is performed in each memeplex using a particle swarm optimization like method. To ensure global exploration, a shuffled information exchange will occur between memeplexes after a defined number of evolution steps. Infection of a memeplex's frogs by the frogs from other memeplexes ensures the improvement of frog ideas quality and that the cultural evolution towards any particular interest is unbiased. Moreover, if the local search cannot find better solutions, random virtual frogs are generated and substituted in the population. Then local search and shuffling processes (global relocation) will continue until defined convergence criteria are satisfied. The flowchart diagram of the SFLA is shown in Fig. 1.

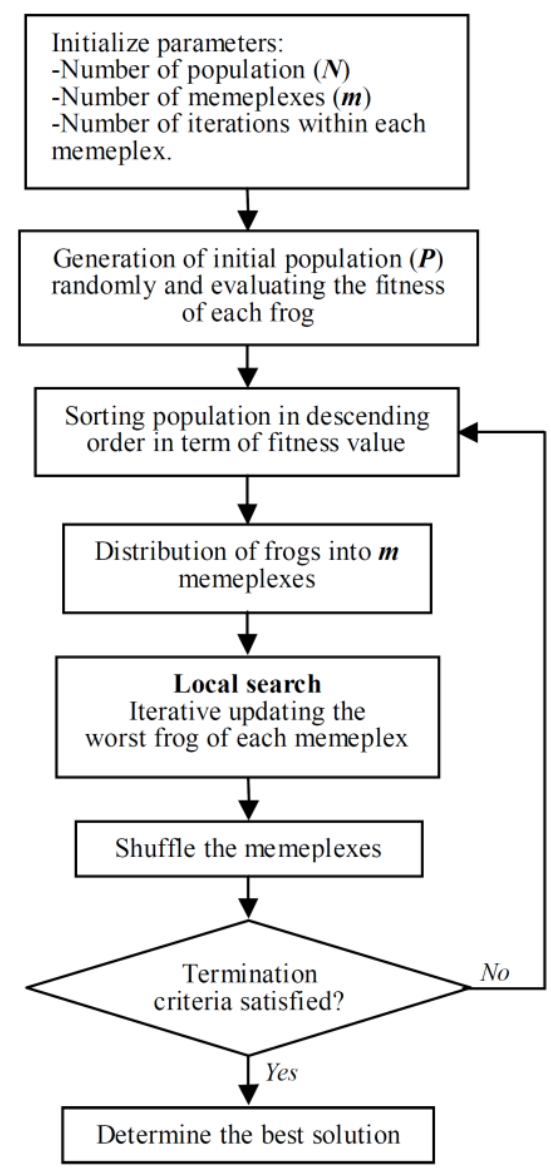

Fig. 1. General principle of SFLA.

The SFLA begins with an initial population of " $N$ " frogs $P=\left\{X_{1}, X_{2}, \ldots, X_{N}\right\}$ which are created randomly within the feasible space $\Omega$. For $S$-dimensional problems ( $S$ variables), the position of the $i$ th frog is represented as $X_{i}=\left[x_{i 1}, x_{i 2}, \ldots, x_{i s}\right]^{T}$. to evaluate the frog's position, a fitness function is defined.
Then the performance of each frog is computed based on its position. The frogs are sorted in a descending order regarding to their fitness. Then, the entire population is divided into $m$ memeplexes, each of which consisting of $n$ frogs (i.e. $N=n \times m)$. The division is done by distributing the frogs one by one and in order between the $m$ existing memeplexes.

Within each memeplex, the position of frog $i$ th $\left(D_{i}\right)$ is adjusted according to the difference between the frog with the worst fitness $\left(X_{w}\right)$ and the frog with the best fitness $\left(X_{b}\right)$ as shown in (13), where rand () is a random number in the range of $[0,1]$. During memeplex evolution, the worst frog $X_{w}$ leaps toward the best frog $X_{b}$. According to the original frog leaping rule, the position of the worst frog is updated as follow:

$$
\begin{gathered}
\text { Position change }\left(\mathrm{F}_{\mathrm{j}}\right)=\operatorname{rand}() \times\left(Z_{b}-Z_{w}\right) . \\
\qquad Z_{w}(\text { new })=Z_{w}+F,\left(\|F\|<F_{\max }\right) .
\end{gathered}
$$

where $D_{\max }$ is the maximum allowed change of frog's position in a single jump. If a frog with a better fitness value is produced in this process, it replaces the worst frog, otherwise, the calculation in (13) and (14) are repeated with respect to the global best frog $\left(X_{g}\right)$, (i.e. $X_{g}$ replaces $X_{b}$ ). If no improvement becomes possible in this case, then a new frog is randomly generated to replace the worst frog. The evolution process will continue for a specific number of iterations [6].

\section{B. String Encoding and Mapping Procedure}

In this study, integer string structure and mapping procedure used in [5] were applied to encode a solution of the problem so that, both SFLA and GA methods can be implemented. State vector, $X_{t}$, and decision vector, $U_{t}$, have dimension of MW, but it can be converted into vectors which have information on the number of units in each unit type. This mapping procedure is very useful for SFLA and GA implementation of a GEP problem such as encoding and treatment of inequality (9).

The length of a string is equal to the product of the number of planning stages and the number of candidate unit's types. Fig. 2 illustrates a five type, three stage example of a string in which each string position represents the number of candidate units in each type to be constructed in each stage of planning horizon. For instance, the first three numbers, in this case, 2, 5 and 0 represents the number of candidate units of type 1 in stages 1 through 3 of planning horizon.

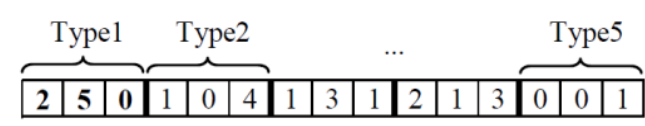

Fig. 2. A five type, three stage example of a string.

\section{Generation of Initial String Population}

An initial population is randomly generated so that inequality (9) is satisfied. Therefore, for each string position, values representing the number of candidate units in each stage for every particular type, is randomly generated using a uniform distribution between $\left[0, U_{\max }\right]$, where $U_{\max }$ is the upper construction limit of candidate units. 


\section{Evaluation of fitness function}

The fitness function used here is the total cost over the planning horizon represented by objective function (1) plus penalized cost of infeasibility. Before evaluation, all strings are checked for feasibility by constraints (10) and (11). If any string violates over (10) and (11), only the parts of the string that violate the constraints in stage $t$ are regenerated at random until they satisfy the constraints. The concept of penalty function is used to penalize strings which fail to satisfy reliability criteria of (12). The penalty function for $n$th string is set as follows:

$$
\operatorname{Penalty}(n)=\left\{\begin{array}{cc}
p \times \bar{O}\left(X_{t}\right) & \text { LOLP }>\varepsilon \\
0 & \text { oth. }
\end{array}\right.
$$

The penalty factor, $p$ is a large value. Consequently, using (1), the fitness value of string $n$ is:

$$
\text { fitness }(n)=\left\{\begin{array}{cc}
C(n) & \text { if } n \text { is feasible } \\
C(n)+\text { Penalty }(n) & \text { oth. }
\end{array}\right.
$$

The best string is the one that has the minimum fitness value.

\section{TEST REsUlts}

The SFLA and GA were implemented using MATLAB code. The test system's description, parameters of GEP and SFLA and the results for each case study are presented in the following sections respectively.

\section{A. Test System Description}

The forecasted peak load and other data of a test system with 15 existing power plants and five types of candidate options are taken from [5]. The SFLA and GA methods have been applied to this system with 2 planning horizons: case 1 with a 10-year planning period and case 2 for a real-scale system with a 20 -year planning period. Each planning horizon consists of two-year stages, making them to be five and ten stages in case 1 and case 2, respectively. Number of years between the reference date of cost calculations and the first year of study $(t o)$ is assumed to be 2 years. The forecasted peak demand over the study period is shown in Table I. Technical and economical data of existing and candidate plant options for future addition is given in Tables II and III, respectively.

\section{B. Parameter for GEP and SFLA}

In this paper, we use $8.5 \%$ as discount rate, 1 day per year $(0.27 \%)$ as LOLP criteria in each stage, and the lower and upper bounds for reserve margin are set at $20 \%$ and $50 \%$ respectively. EENS cost is assumed to be $0.05 \$ / \mathrm{kWh}$. The lower and upper bounds of capacity mixes by fuel types are $0 \%$ and $30 \%$ for oil-fired power plants, $0 \%$ and $40 \%$ for LNG-fired, $20 \%$ and $60 \%$ for coal-fired, and $30 \%$ and $60 \%$ for nuclear, respectively. Salvage factor $(\delta)$ is calculated by sinking fund depreciation method [2].

Since the SFLA parameters have significant effect on the quality of solution, they were evaluated before commencing this experiment for the suitable parameter values of population size, number of iterations, number of memeplexes, and $D_{\max }$. As a result, in Table IV their selected values to be used in our SFLA experiment are shown. Moreover, based on many trials, the penalty factor, $p$ of $1,000,000$ is used to ensure that the infeasible solutions are penalized.

\begin{tabular}{l|c|c|c|c|c|c}
\multicolumn{7}{c}{ TABLE I: FORECASTED PEAK DEMAND [5] } \\
\hline Stage (year) & 0 & 1 & 2 & 3 & 4 & 5 \\
\hline Peak Demand (MW) & 5000 & 7000 & 9000 & 10000 & 12000 & 13000 \\
\hline Stage (year) & - & 6 & 7 & 8 & 9 & 10 \\
\hline Peak Demand(MW) & - & 14000 & 15000 & 17000 & 18000 & 20000 \\
\hline
\end{tabular}

TABLE II: TECHNICAL AND ECONOMICAL DATA OF EXISTING PLANTS [5]

\begin{tabular}{l|c|c|c|c|c}
\hline $\begin{array}{c}\text { Name } \\
\text { (fuel Type) }\end{array}$ & $\begin{array}{c}\text { No. of } \\
\text { Units }\end{array}$ & $\begin{array}{c}\text { Unit } \\
\text { Capacity } \\
(\mathrm{MW})\end{array}$ & $\begin{array}{c}\text { FOR } \\
(\%)\end{array}$ & $\begin{array}{c}\text { Operating } \\
\text { Cost } \\
(\$ / \mathrm{kWh})\end{array}$ & $\begin{array}{c}\text { Fixed O\&M } \\
\text { Cost } \\
(\$ / \mathrm{kW}-\mathrm{Mon})\end{array}$ \\
\hline Oil \#1 (Heavy Oil) & 1 & 200 & 7.0 & 0.024 & 2.25 \\
\hline Oil \#2 (Heavy Oil) & 1 & 200 & 6.8 & 0.027 & 2.25 \\
\hline Oil \#3 (Heavy Oil) & 1 & 150 & 6.0 & 0.030 & 2.13 \\
\hline LNG G/T \#1 (LNG) & 3 & 50 & 3.0 & 0.043 & 4.52 \\
\hline LNG C/C \#1 (LNG) & 1 & 400 & 10.0 & 0.038 & 1.63 \\
\hline LNG C/C \#3 (LNG) & 1 & 400 & 10.0 & 0.040 & 1.63 \\
\hline LNG C/C \#4 (LNG) & 1 & 450 & 11.0 & 0.035 & 2.00 \\
\hline Coal \#1 (Anthracite) & 2 & 250 & 15.0 & 0.023 & 6.65 \\
\hline Coal \#2 (Bituminous) & 1 & 500 & 9.0 & 0.019 & 2.81 \\
\hline Coal \#3 (Bituminous) & 1 & 500 & 8.5 & 0.015 & 2.81 \\
\hline Nuclear \#1 (PWR) & 1 & 1000 & 9.0 & 0.005 & 4.94 \\
\hline Nuclear \#2 (PWR) & 1 & 1000 & 8.8 & 0.005 & 4.63 \\
\hline
\end{tabular}

TABLE III: TECHNICAL AND ECONOMICAL DATA OF CANDIDATE PLANTS [5]

\begin{tabular}{l|c|c|c|c|c|c|c}
\hline $\begin{array}{c}\text { Candidate } \\
\text { Type }\end{array}$ & $\begin{array}{c}\text { Const. Upper } \\
\text { Limit }\end{array}$ & $\begin{array}{c}\text { Capa- } \\
\text { city } \\
(\mathrm{MW})\end{array}$ & $\begin{array}{c}\text { FOR } \\
(\%)\end{array}$ & $\begin{array}{c}\text { Operating } \\
\text { Cost } \\
(\$ / \mathrm{kWh})\end{array}$ & $\begin{array}{c}\text { Fixed } \\
\text { O\&M } \\
\text { Cost }\end{array}$ & $\begin{array}{c}\text { Capital } \\
\text { Cost } \\
(\$ / \mathrm{kW})\end{array}$ & $\begin{array}{c}\text { Life } \\
\text { Time }\end{array}$ \\
\hline Oil & 5 & 200 & 7.0 & 0.021 & 2.20 & 812.5 & 25 \\
\hline LNG C/C & 4 & 450 & 10.0 & 0.035 & 0.90 & 500.0 & 20 \\
\hline Coal (Bit.) & 3 & 500 & 9.5 & 0.014 & 2.75 & 1062.5 & 25 \\
\hline Nuc. (PWR) & 3 & 1000 & 9.0 & 0.004 & 4.60 & 1625.0 & 25 \\
\hline Nuc.(PHWR) & 3 & 700 & 7.0 & 0.003 & 5.50 & 1750.0 & 25 \\
\hline
\end{tabular}

TABLE IV: BEST PARAMETERS FOR SFLA IMPLEMENTATION \begin{tabular}{l|l|l|l|l}
\hline SFLA Parameters & Population size & No. of Iterations & Memplexes & $\mathrm{D}_{\max }$ \\
\hline
\end{tabular} \begin{tabular}{l|l|l|l|l}
\hline Values & 500 & 100 & 10 & Inf. \\
\hline
\end{tabular}

\section{Numerical Results}

Since the SFLA method is random-based, each run may yield a different solution. Therefore, each case study is solved in ten runs and the best solution is then chosen between them. This solution for each case study is shown in Table V. For comparison, each case study is similarly solved by GA method and its results are also reported in Table V. Comparing the results shows the solution quality and accuracy in SFLA are better than GA in both cases. In case 1 and case 2, SFLA has achieved a $0.031 \%$ and $0.15 \%$ improvement in costs over GA, respectively. It should be noted that, the results of the GA method are obtained over 500 iterations. In case 2, performance of SFLA is more evident with increment of decision variables.

Since a long-term GEP problem deal with a great amount of investment, a slight improvement in expansion plan cost by the proposed SFLA method can result in considerable cost saving for electric utilities.

Fig. 3 and Fig. 4, illustrate the convergence characteristics of SFLA and GA methods in case 1 and case 2, respectively. In these figures, the mean values of 10 run characteristics are depicted for 100 iterations for both methods. The SFLA shows better performance than GA in both cases. Table VI summarizes the optimal results obtained by SFLA method for generation expansion plans of case 1 and case 2 which are the 
number of each type of power plants in each stage of planning periods. The results obtained by GA are shown in Table VII for comparison.

\section{CONCLUSIONS}

This paper proposed a new approach based on the shuffled frog leaping algorithm (SFLA) to solve long-term least-cost generation expansion planning problem in power systems. Incorporation of integer encoding, mapping procedure and penalty factor approach improved the proposed method. Integer encoding and mapping procedure made easier implementation of SFLA to the GEP problem especially for construction capability limits to be taken into account. Also, the penalty factor approach, improved effectiveness and efficiency of the SFLA searches where inequality constraints are involved.

The SFLA has been successfully applied to long-term GEP problem with results that outperform GA in term of success rate and solution quality. The proposed SFLA could also achieve an order of magnitude of improvement, especially in larger scale GEP problems. Therefore, it can be employed as a planning tool for long-term generation expansion planning in a real-system scale.

\begin{tabular}{l|c|c}
\multicolumn{2}{c}{ TABLE V: COST OF BEST SOLUTIONS OBTAINED BY SFLA AND GA } \\
\hline \multirow{2}{*}{ Solution Method } & Cumulative Discounted Cost (Million \$) \\
\cline { 2 - 3 } & Case 1 & Case 2 \\
& (10-year study Period) & (20-year study Period) \\
\hline SFLA & $\mathbf{1 0 9 3 7 . 0 1}$ & $\mathbf{1 9 6 8 9 . 8 7}$ \\
\hline GA & 10940.40 & 19719.50 \\
\hline
\end{tabular}

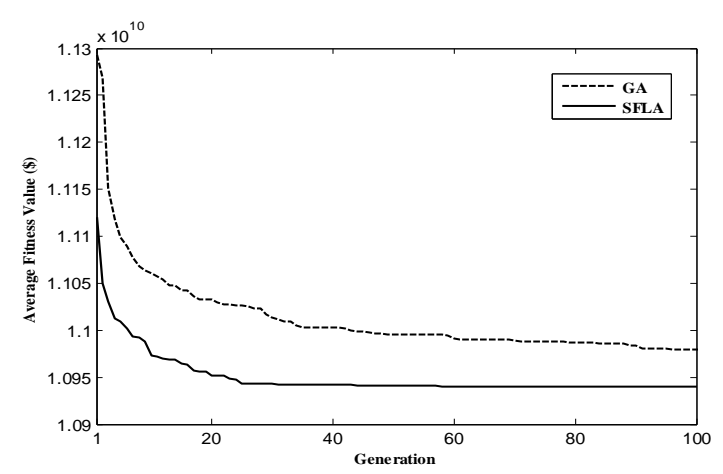

Fig. 3. Convergence characteristics of SFLA and GA methods in Case 1 system.

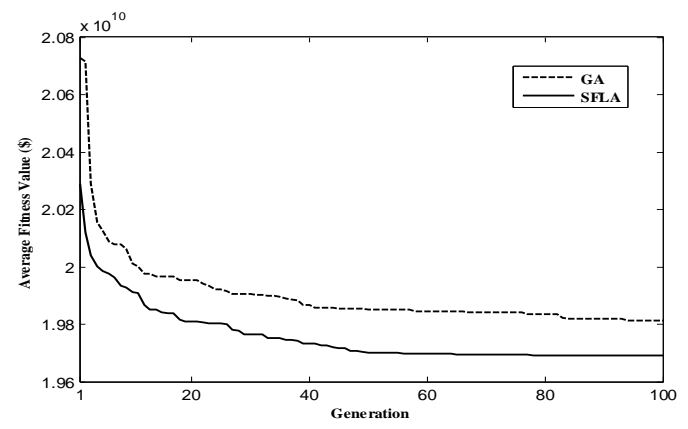

Fig. 4. Convergence characteristics of SFLA and GA methods in Case 2 system.

TABLE VI: Number of NEWLy INTRODUCED Plants In CASE 1 AND CASE 2 BY SFLA METHOD

\begin{tabular}{l|c|c|c|c|c|c|c|c|c|c}
\hline \multirow{2}{*}{$\begin{array}{c}\text { Candidate Type } \\
\text { Stage }\end{array}$} & \multicolumn{7}{|c}{ Number of Unit Selected } \\
\cline { 2 - 12 } & I & II & III & IV & V & VI & VII & VIII & IX & X \\
\hline Oil-200MW & $0(2)^{*}$ & $1(1)$ & $1(1)$ & $4(4)$ & $1(1)$ & 1 & 1 & 3 & 3 & 2 \\
\hline LNG C/C-450MW & $3(3)$ & $2(1)$ & $0(0)$ & $1(1)$ & $1(1)$ & 0 & 1 & 0 & 0 & 1 \\
\hline
\end{tabular}

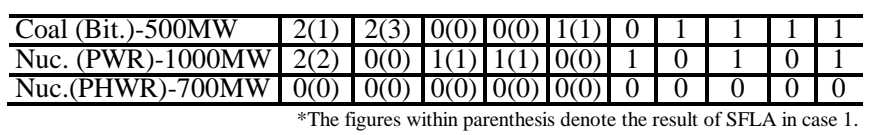

TABLE VII: Number of NEWLy INTROduced Plants In CASE 1 AND CASE

\begin{tabular}{c|c|c|c|c|c|c|c|c|c|c}
\hline \multicolumn{1}{c|}{$\begin{array}{c}\text { Candidate Type } \\
\text { Stage }\end{array}$} & \multicolumn{7}{c}{ Bumber of Unit Selected } \\
\cline { 2 - 12 } & I & II & III & IV & V & VI & VII & VIII & IX & X \\
\hline Oil-200MW & $0(2)^{*}$ & $1(1)$ & $1(1)$ & $0(4)$ & $2(1)$ & 1 & 1 & 4 & 1 & 0 \\
\hline LNG C/C-450MW & $3(3)$ & $2(1)$ & $1(0)$ & $1(1)$ & $0(1)$ & 0 & 0 & 1 & 1 & 0 \\
\hline Coal (Bit.)-500MW & $2(3)$ & $2(1)$ & $1(0)$ & $2(0)$ & $1(1)$ & 0 & 0 & 1 & 1 & 2 \\
\hline Nuc. (PWR)-1000MW & $2(1)$ & $0(1)$ & $0(1)$ & $1(1)$ & $0(0)$ & 1 & 1 & 0 & 0 & 1 \\
\hline Nuc.(PHWR)-700MW & $0(0)$ & $0(0)$ & $0(0)$ & $0(0)$ & $0(0)$ & 0 & 0 & 1 & 0 & 0 \\
\hline
\end{tabular}

*The figures within parenthesis denote the result of GA in case 1 .

\section{REFERENCES}

[1] A. G. Kagiannas, D. T. Askounis, and J. Psarras, "Power generation planning:A survey from monopoly to competition," Int. J. Elect. Power Energy Syst. vol. 26, no. 6, pp. 413-421, 2004.

[2] Internatinal Atomic Energy Agency, "Wien Automatic System Planning (WASP) package - A computer code for power generating system expansion planning, version WASP-IV user's manual," IAEA, Vienna, 2006

[3] J. Zhu and M. Y. Chow, "A review of emerging techniques on generation expansion planning," IEEE Trans. Power Systems, vol. 12, no. 4, pp. 1722-1728, Nov. 1997.

[4] S. Kannan, S. M. R. Slochanal, and N. P. Padhy, "Application and comparison of metaheuristic techniques to generation expansion planning problem," IEEE Trans. on Power Systems, vol. 20, no. 1, pp. 466-475, Feb. 2005.

[5] J. B. Park, Y. M. Park, J. R. Won, and K. Y. Lee, "An improved genetic algorithm for generation expansion planning," IEEE Trans. on Power systems, vol. 15, no. 3, pp. 916-922, Aug. 2000.

[6] M. M. Eusuff, K. Lansey, and F. Pasha, "Shuffled frog-leaping algorithm: a memetic meta-heuristic for discrete optimization," Engineering Optimization, vol. 38, no. 2, pp.129-154, 2006.

[7] J. Ebrahimi, S. H. Hosseinian, and G. B. Gharehpetian, "Unit commitment problem solution using shuffled frog leaping algorithm," IEEE Trans. on Power systems, vol. 26, no. 2, pp. 573-581, 2011.

[8] G. Chen, J. Chen, and X. Duan, "Power flow and dynamic optimal power flow including wind farms," in Proc. 1th International Conference on Sustainable Power Generation and Supply, Nanjing, China, 2009, pp. 1-6.

[9] Q. Li, "Shuffled frog leaping algorithm based optimal reactive power flow," presented at the International Symposium on Computer Network and Multimedia Technology, Wuhan, China, December 18-20, 2009.

[10] Y. Fukuyama and H. D. Chiang, "A parallel genetic algorithm for generation expansion planning," IEEE Trans. on Power Systems, vol 11, no. 2, pp. 955-961, 1996.

[11] J. Sirikum and A. Techanitisawad, "Power generation expansion planning with emission control: a nonlinear model and a GA-based heuristic approach," Int. J. Energy Res. vol. 30, pp. 81-99, 2006.

[12] X. Wang and J. R. McDonald, Modern power system planning, McGraw-Hill, 1994, ch. 3, pp. 208-229.

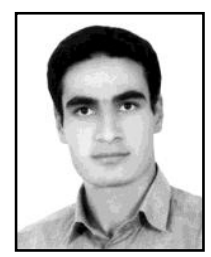

Morteza Jadidoleslam received the B.Sc. degree in Electrical Engineering from Kerman University, Kerman, Iran in 2008. He received the M.Sc. degree from Isfahan University of Technology (IUT), Isfahan, Iran in 2011. Currently, he is pursuing his Ph.D. at the Isfahan University of Technology (IUT), Isfahan, Iran His research interests include power system operation and planning, generation expansion planning, computational intelligence and their applications to power systems.

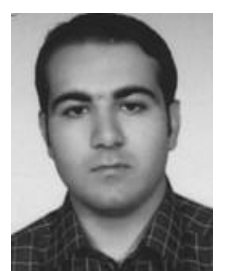

Ehsan Bijami received his B.S. degree in Electrical Engineering from Kerman University, Iran in 2008. From 2008 to 2010 he was as postgraduate student in Isfahan University of Technology , Isfahan, Iran, where he received M.Sc. degree on Control Engineering. He is currently spending the military Servisce period. His interests include power system control and stability, soft computing, renewable energy, robust control and model predictive control. 


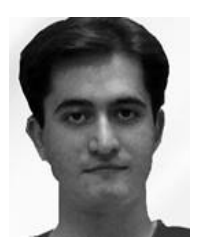

Navid Amiri received the B.Sc. and the M.Sc. degrees in electrical engineering from Isfahan University of Technology (IUT), Isfahan, Iran, in 2008 and 2011, respectively. Currently, he is pursuing his Ph.D. at the Iran University of Science and Technology Tehran, Iran. His research interests include nonlinear control, fault diagnosis in electromechanical systems, power electronics, and variable-speed ac drives.

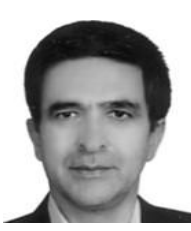

Akbar Ebrahimi received the B.Sc. degree from Amir Kabir University, Tehran, Iran, in 1979, M.Sc. degree from University of Manchester, Institute of Science and Technology (UMIST), Manchester, U.K. in 1985, and Ph.D. Degree from Tarbiat Modares University, Tehran, in 1994, all in electrical power engineering. Currently, he is an assistant professor at Isfahan University of Technology, Isfahan, Iran. His research interests are power system operation, reliability evaluation, and planning with applications of artificial intelligence and Bayesian analysis.

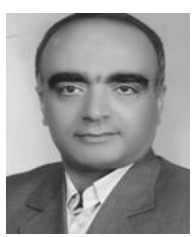

Javad Askari-Marnani received the B.Sc. and M.Sc. degrees in electrical engineering from Isfahan University of Technology in 1987 and from University of Tehran in 1993, respectively. He received also Ph.D degrees in electrical engineering from University of Tehran in 2001. From 1999 to 2001, he received a grant from DAAD and joined Control Engineering department at Technical University Hamburg - Harburg in Germany, where he completes his Ph.D. with Professor Lunze's research group. He is currently an assistant professor at control engineering department of Isfahan University of Technology. His current research interests are in control theory, particularly in the field of Hybrid Dynamical Systems and Fault-Tolerant Control, Identification, Discrete-Event Systems, Graph Theory and Electrical Engineering Curriculum. 